\title{
"Do You Know What I Know?": How Communication Norms and Recipient Design Shape the Content and Effectiveness of Patient Handoffs
}

\author{
Nicholas A. Rattray, $P h D^{1,2,3}$, Mindy E. Flanagan, $P h D^{7}$, Laura G. Militello, $M A^{4}$, \\ Paul Barach, MD, MPH ${ }^{5}$, Zamal Franks, BS ${ }^{7}$, Patricia Ebright, RN, PhD, FAAN', \\ Shakailb U. Rehman, $M D^{7}$, Howard S. Gordon, $M D^{8}$, and Richard M. Frankel, PhD 13,3
}

\begin{abstract}
'VA HSR\&D Center for Health Information and Communication, Roudebush VAMC, Indianapolis, USA; ${ }^{2}$ Department of Anthropology, Indiana University-Purdue University Indianapolis, Indianapolis, USA; ${ }^{3}$ Regenstrief Institute, Inc., Indianapolis, IN, USA; ${ }^{4}$ Applied Decision Science, LLC, Dayton, USA; ${ }^{5}$ Jefferson College of Population Health, Wayne State University School of Medicine, Detroit, USA; 9 Indiana University School of Nursing, Indianapolis, USA; 'P Phoenix VA Healthcare Systems, University of Arizona College of Medicine-Phoenix, Phoenix, USA; ${ }^{8}$ Jesse Brown VAMC, VA HSR\&D Center of Innovation for Complex Chronic Healthcare, University of Illinois at Chicago, Chicago, USA; ' Indiana University School of Medicine, Indianapolis, USA.
\end{abstract}

BACKGROUND: Poor communication during end-of-shift transfers of care (handoffs) is associated with safety risks and patient harm. Despite the common perception that handoffs are largely a one-way transfer of information, researchers have documented that they are complex interactions, guided by implicit social norms and mental frameworks.

OBJECTIVES: We investigated communication strategies that resident physicians report deploying to tailor information during face-to-face handoffs that are often based on their implicit inferences about the perceived information needs and potential harm to patients.

METHODS/PARTICIPANTS: We interviewed 35 residents in Medicine and Surgery wards at three VA Medical Centers (VAMCs).

MAIN MEASURES: We conducted qualitative interviews using audio-recorded semi-structured cognitive task interviews.

KEY RESULTS: The effectiveness of handoff communication depends upon three factors: receiver characteristics, type of shift, and patient's condition and perceived acuity. Receiver characteristics, including subjective perceptions about an incoming resident's training or ability levels and their assumed preferences for information (e.g., detailed/ comprehensive vs. minimal/"big picture"), influenced content shared during handoffs. Residents handing off to the night team provided more information about patients' medical histories and care plans than residents handing off to the day team, and higher patient acuity merited more detailed information and the medical service(s) involved dictated the types of information conveyed.

CONCLUSIONS: We found that handoff communication involves a complex combination of socio-technical information where residents balance relational factors against

Electronic supplementary material The online version of this article (https://doi.org/10.1007/s11606-018-4755-5) contains supplementary material, which is available to authorized users.

Received November 28, 2017

Revised May 30, 2018

Accepted November 9, 2018

Published online December 10, 2018 content and risk. It is not a mechanistic process of merely transferring clinical data but rather is based on learned habits of communication that are context-sensitive and variable, what we refer to as "recipient design." Interventions should focus on raising awareness of times when information is omitted, customized, or expanded based on implicit judgments, the emerging threats such judgments pose to patient care and quality, and the competencies needed to be more explicit in handoff interactions.

KEY WORDS: communication; resident handoffs; qualitative research; sociolinguistics; quality of care; patient safety; risk management.

J Gen Intern Med 34(2):264-71

DOI: $10.1007 / \mathrm{s} 11606-018-4755-5$

(c) Society of General InternalMedicine (This is a U.S. government work and not under copyright protection in the U.S.; foreign copyright protection may apply) 2018

\section{INTRODUCTION}

Communication breakdowns stemming from omitted or inaccurate information, failure to follow best practices, and persistent interruptions are associated with adverse events across patient care settings. ${ }^{1-5}$ Care that involves multiple transfers of responsibility, such as end-of-shift handoffs from one provider to another, represents one source of error that can adversely affect patient safety and one that is highly shaped by the micro culture of the organization in which they occur. ${ }^{6,7}$ Handoffs can be defined as "the exchange between health professionals of information about a patient accompanying either a transfer of control over, or of responsibility for, the patient." ${ }^{\text {" Handoff communica- }}$ tion involves complex face-to-face interactions that are coconstructed by participants based on shared social norms and implicit assumptions and inferences about receivers and senders. Rather than being mere technical transfers of clinical information, ${ }^{9}, 10$ handoffs are multifunctional in simultaneously accomplishing several objectives including transmission of technical patient information, transferring legal responsibility for 
patients, maintaining continuity of care, and educating trainees. ${ }^{11}$ At the same time, they resist standardization, ${ }^{12}$ in part, because they are constrained by structural and human factors such as the time of day or night, variations in the physical environments in which they occur, availability of resources such as computers and electronic health records, and the micro culture of the organization. ${ }^{7,} 13$ Improvements in handoff communication must accommodate attempts to harmonize work flow routines while simultaneously encouraging agile adjustments to shifting contexts and situational demands on providers. ${ }^{11}$

Handoff encounters can be seen as "naturally occurring" instances of social interaction and therefore of interest to those who study language as well as clinicians. Sociolinguistics is the study of language in the context of social interaction. It focuses on the linguistic competencies speakers use to communicate effectively with one another. For example, while a physician might characterize a patient's symptoms in talking to a fellow physician as "trigeminal neuralgia," she would likely use the term "facial pain" in talking with the patient, a shift known as a change in register. ${ }^{14}$ Adapting one's language during a joint task, like a handoff where information needs to vary, requires a complex set of learned interactional skills. Sociolinguistic approaches have been used to explore how physicians adapt their communication depending on who is receiving the message and their perceived communication needs. ${ }^{15-17}$ We refer to the skilled use of language in context to shape an action for an intended recipient as "recipient design." Recipient design is a technical term referring to any shaping of an action for an intended recipient. In conversations between doctors and patients, it focuses attention on the competences, knowledge, and situational awareness each of the interactants uses to demonstrate understanding and to make themselves understood. ${ }^{18,19}$ While most studies of recipient design have focused on patient-provider interactions, the framework offers a useful way to understand information tailoring during complex physician conversational events such as handoffs. ${ }^{20,21}$ Few studies have addressed the issue of how providers tailor their handoff approaches to particular settings. ${ }^{22-24}$

This study aimed to gain insights into the cognitive strategies that guide resident physicians' handoff preparation, enactment, and post-handoff activities. While prior research has identified gaps in communication during handoffs, there is little research that explores the cognitive and contextual factors that shape these exchanges. Cognitive task analysis methods include interview techniques designed specifically to unpack these often difficult-to-articulate aspects of work. $^{25-27}$ We used cognitive interviews to explore how residents structure their thoughts and communication when they handoff patients, and what contextual and interpersonal factors influence how they tailor information "on the fly" to meet the presumed needs of the receiving physician. A sociolinguistic framework and individual CTA interviews provide valuable insights into the underlying shared values, beliefs, assumptions, and norms of health care providers. Understanding these thought processes offers a unique glimpse into how and why handoffs either succeed or contribute to medical errors.

\section{Setting and Participants}

METHODS

We conducted a prospective, qualitative study that included cognitive task interviews at three VAMCs located in different states. The US Department of Veterans Affairs provides fertile ground for in-depth qualitative study as the largest integrated health provider in North America, and through its efforts at quality improvement. We identified key informants at each site from the departments of Medicine and Surgery to participate in interviews. Key informants are typically "knowledgeable insiders" who are able to share useful information about a phenomenon of interest. Because of their unique position in two worlds, they are often thought of as "cultural go-betweens."28, 29 Key informants were recommended by program directors or chief residents at the three sites. Residents were asked to identify colleagues like themselves, a technique known as "snowball sampling."30 Key informants received a $\$ 100$ gift card. Ethics approval was obtained from the University Institutional Review Board and the VAMC R\&D Human Subjects board.

\section{Data Collection}

We conducted semi-structured individual interviews using CTA to understand resident thought processes before, during, and after a shift handoff. CTA methods ${ }^{31-34}$ are designed to aid experienced practitioners in articulating cognitive aspects of work that are typically difficult to express. Grounding the interview in a lived incident supports recall of specific cues directly sensed by interviews (i.e, seen, heard, etc.) and how the interviewee interpreted and acted on the cues. ${ }^{25,26}$ The interviews were adapted to explore strategies that experienced residents used in tailoring handoff interactions. Data were collected during separate site visits from April 2015 through June 2016. A CTA expert (LGM) trained two research assistants (RAs) by practicing interviews and offering feedback. The RAs conducted 1-h semi-structured interviews with each resident, adapting two well-established knowledge elicitation methods. First, in the Task Diagram portion of the interview, residents were asked to describe the major steps in preparing for a handoff. ${ }^{27}$ Second, using an adaptation of the Critical Decision Method interview, residents were asked to recall their last handoff (see online Appendix Table 1 for the interview guide). ${ }^{26}$ The research team adapted a series of critical decision method questions and probes to elicit strategies, goals, and types of information shared during handoff interactions. Interviewers received feedback on the first five interviews (from authors PE and LGM). Care was taken to include participants who received and gave handoffs and whose last handoff was day-to-night as well as those whose handoff was on night-to-day shift. The interviewers also asked about formal or informal training and first-hand experiences in supervising handoffs. Residents involved in handoffs either in the Medicine wards or in the Surgical services were contacted and 
consented prior to conducting the interview. Notably, 32 of the 35 interviews were "fresh" having been conducted within 10 min of a completed handoff; the remaining three were conducted within $48 \mathrm{~h}$. The interviews were conducted in a quiet location near the hospital's patient ward. They were audio-recorded and transcribed verbatim according to a standardized format. Team members compared the recordings with the written transcripts to ensure accuracy.

\section{Data Analysis}

Interview materials were analyzed using thematic analysis ${ }^{35}$ and immersion/crystallization methods. ${ }^{36}$ Thematic analysis is commonly used with cognitive task analysis data to encourage insight and discovery by assembling analysts with different perspectives to review and organize data into emergent categories and meaningful themes. ${ }^{26}$ Immersion-crystallization techniques were employed to draw on the clinical insights of the larger team through repeated readings of interviews. A multidisciplinary team including clinicians analyzed the transcripts by developing a qualitative codebook and applying codes to interview excerpts through an iterative, consensusbased approach. All team members independently reviewed at least $25 \%$ of transcripts. Each analyst identified text that involved tailoring handoff information in "open coding." After comparing provisional codes, differences were reconciled to build an inventory of codes. A confirmability audit to ensure dependability of the analysis involved matching code definitions with transcripts. ${ }^{37}$ Results of the preliminary analysis were shared with study investigators uninvolved in the initial analysis to detect potential bias and propose alternative interpretations using conference calls and email correspondence. Themes related to adjustments in the amount and type of information such as familiarity with patient, patient status, receiver past performance, receiver preferences for information, receiver training level, type of service, and shift type shared during handoffs were considered as recipient design.

The entire corpus of interviews was coded using qualitative software NVivo 10; one member of the data analysis team (NR) was responsible for updating and maintaining a master file of the coded transcripts. Multiple reviews of the data were used to identify key categories. Investigators reviewed data in each category, extracting themes and iteratively discussing them with the analysis team until consensus was reached. ${ }^{38}$ Comparisons were made by site, resident type, and type of service (Medicine and Surgery).

\section{RESULTS}

A total of thirty-five residents across the three sites participated in the CTA interviews (see Table 1). The data analysis resulted in 7 categories from which 3 themes emerged: (I) receiver characteristics, (II) type of shift and local practice, and (III) patient acuity and receiving resident familiarity with patients (see online Appendix 2).

\section{Theme I: Receiver Characteristics: Reputation and Past Performance Preferences, and Training}

Three categories emerged within this theme, Reputation and/ or Perception of Past Performance, Perceived Receiver preferences, Training Level. Analysis of the interview data revealed that residents handing off patients rarely followed a standardized format.

(a) Reputation or Perception of Past Performance. Residents reported evaluating other residents' clinical competency subjectively, either through their own interactions or through hearsay from others, and applying that information to tailor their handoffs. Specifically, in cases where a resident was deemed to have a "good reputation and clinical judgment," the outgoing day shift resident might abbreviate the handoff.

Residents made snap judgments about another resident's competence during the handoff. For example, when the receiving resident was not paying close attention, decreased trust resulted, leading to an outgoing resident querying the receiving resident about upcoming tasks. One resident explained: "If they are somebody who we really trust ..., I feel a little bit better. If somebody is a little bit less so, I give a lot more instruction." In other cases, receiving residents asked questions to demonstrate their understanding and planning. One resident described an unsatisfactory encounter: "The sign-out that I got ... was more frustrating because I don't feel like they're giving me all of the tools that I need to safely take care of the patient ... I ask more questions. I say, why are they here? Why did they present in the first place?"

(b) Perceived Receiver preferences. Residents altered the amount and format of patient information included in the handoff based on the receiving resident's presumed preference for detailed versus brief accounts. For receivers known to prefer high-level patient information, outgoing residents focused on tasks that needed to be completed for the most unstable patients. In these handoffs, "routine" patient information was typically omitted. Only one resident explicitly reported asking receiving residents whether they preferred a more or less detailed handoff; the remaining interviewees used indirect means of discerning incoming residents' preferences. Importantly, patterns of attunement to individual residents' preferences were established and maintained over the course of a rotation, typically without any explicit agreement. Another resident described how they adjusted their presentation of information: "I mean if there is something that I know that somebody wants to know extra or wants highlighted on every patient because it makes their comfort level better, then I'll do that." Residents varied in the extent to which they engaged in social talk as well as more 
Table 1 Interviews Analyzed for Handoff: Characteristics: Care Providers and Study Sites

\begin{tabular}{|c|c|c|c|c|c|c|c|}
\hline & \multicolumn{2}{|l|}{ PGY1 } & \multicolumn{2}{|l|}{ PGY2 } & \multicolumn{2}{|l|}{ PGY3 } & \multirow[t]{2}{*}{ Total } \\
\hline & Medical & Surgical & Medical & Surgical & Medical & Surgical & \\
\hline Site 1 & 0 & 0 & 7 & 1 & 1 & 0 & 9 \\
\hline Site 2 & 12 & 2 & 1 & 2 & 0 & 1 & 18 \\
\hline Site 3 & 8 & 0 & 0 & 0 & 0 & 0 & 8 \\
\hline Total & 20 & 2 & 8 & 3 & 1 & 1 & 35 \\
\hline
\end{tabular}

technical exchanges about transferring patient care based on their perception of resident preferences.

(c) Training Level. Study participants discussed how they tailored communication content according to the recipient's training level. Residents adapted handoffs to include more patient details and instructions when the recipient was an intern (post-graduate year 1), and especially at the beginning of their internship year. Participants suggested that trainees were still developing clinical judgment skills as beginning interns. Handoffs provided an opportunity for senior residents to help interns organize and contextualize medical information, understand information importance, interpret clinical findings, and select interventions. During handoffs to interns, residents reported presenting information slowly, often using repetition, and assessing the recipient's understanding as they went along. Part of this learning process included becoming familiar with specific language and abbreviations used as short-hand descriptions for patient conditions. In contrast, senior residents were viewed as more clinically knowledgeable, able to synthesize clinical information and quickly make recommendations, and able to identify additional patient needs from notes, nurses, and patients after the handoff. As one resident explained, "I think there is a lot of hidden language in sign-out, the way you describe a patient to somebody else." For example, residents discussed how "hidden" language could include body language, such as in cases where outgoing residents explicitly made eye contact and wrote notes on the written sign-out sheet to emphasize the needs of highacuity patients. Compared to handoffs with interns, handoffs to senior residents were characterized by their brevity. Finally, with practice, residents developed efficient approaches to sharing information, such as devoting little or no time to patients who were stable and familiar to the incoming resident, preferring instead to focus on high acuity and patients new to the receiving resident.

\section{Theme II: Type of Shift and Local Practice Context}

(a) Night to Day vs Day to Night Shifts. Residents stressed the importance of offering those on "night float" specific guidelines about larger goals but letting them work out the details on their own. Since night float team residents covered 4 to 5 times as many patients as the day team, opportunities for engagement and expectations for the care were viewed quite differently. For instance, one resident explained that since night floats were often unaware or might forget treatment goals if they were communicated verbally, it was best to write down specific targets for medications or procedures. Some residents suggested that new and unplanned night-time interventions could interfere with the overall care plan, and thus, instructions should be given to minimize these changes to the standing management plan unless the patient deteriorated. Several residents tried anticipating acute events using explicit lists with checkboxes rather than leave to the night float to decide, emphasizing their goal of reducing ambiguity and changes to the care plan.

Residents handing off patients from night to day shifts emphasized concision, and the importance of communicating issues unavailable in the medical record or the rationale for any unplanned treatment ordered overnight. Several notable patterns characterized night to day handoff communications. First, outgoing night residents focused primarily on acute patient issues and changes that occurred during the night shift. When in the role of an incoming day resident, participants explained that they were generally familiar with their own patients and, in some cases, had reviewed the patient record prior to handoff. As a result, this information was typically not discussed or abbreviated during the handoff. However, residents reported that night to day handoffs involved more questions from the day team. Importantly, when patients were admitted overnight, handoff communication conformed more to the approach used in day to night handoffs; it was more complete, included a detailed medical history, and included a triage plan.

A third type of handoff involved residents who were responsible for a large number of patients in the interim between day and night shifts. In this interim period, a double-handoff occurred where a day team resident transferred patients to a different day team resident for approximately $2 \mathrm{~h}$, who then transferred patients to a night float. Among 4 teams at facility 3 , one resident explained how one "call team" covered patients from 5 to $7 \mathrm{pm}$ : "Three of [the teams] leave at $5 \mathrm{pm}$ and one will leave at $7 \mathrm{pm}$, so what you get is what we call a double changeover." Because residents involved in the double 
handoff are not familiar with their patients, providing details written on the handoff form required additional preparation. Additionally, the quality of handoff communication from the cross-cover resident to the night float resident varied considerably. In practice, one person suggested that cross-covering residents, "may or may not pay that much attention to it because they're only going to be cross covering these patients for a couple of hours." Another person shared that certain residents were known to be "great at cross cover," and that trust was built when "you can tell that the cross cover person is really listening and taking care of your patients." Great crosscover residents were well prepared, listened carefully, asked questions, and treated patients from other teams as they would their own patient panel.

(b) Local practice context. Residents discussed adapting handoffs based on the patients' physical location on a unique hospital service and the receiving resident's specialty training. The outgoing resident included patient information that fit with the receiving resident's medical background (and perceived focus). Residents described adapting handoffs to focus on vascular, surgical, and oncology-related concerns. Patients in the intensive care unit were described as having specific information that would align with progress in the patient's care plan. Finally, handoffs between residents with the same training background were described as being briefer due to their shared mental models about specific medical conditions and treatments. One surgery resident described how he came to trust some people over others: "there are just some people that get it. You don't need to spend a lot of time discussing the details of their surgery because they understand."

\section{Theme III: Patient Acuity and Receiving Resident Familiarity with Patients}

(a) Level of Acuity. Residents consistently noted that they adapted their handoff strategy to match their patients' conditions and medical acuity. The type of information that was included in the handoff was specific to the patient's issues (i.e., antibiotics for an infection, obtaining a culture for a patient with fever). Additionally, residents spent more time discussing and providing recommendations for acutely sick patients with high potential to deteriorate. The residents shared their medical history, events during hospitalization, recent active issues, anticipatory instructions for worsening conditions (i.e., abnormal results, changes in status), and follow-up tasks. Handoffs of acute patients might be adapted further based on the receiving resident having less experience caring for such patients. Adaptations included providing education to the receiving resident, having a senior resident present, and ensuring time for clarifying questions. (b) Receiving residents' familiarity with patients. Participants reported adapting handoffs to include more information for receiving residents about new and unfamiliar patients. For example, when beginning a new rotation (e.g., night shift, medical service), residents are not familiar with their patients. As the rotation progresses, they develop familiarity and the primary concerns and priorities for each. As a receiving resident's familiarity with patients grows, outgoing residents reported including less information and providing "more of an update" based on the implicit assumption of a shared mental model. ${ }^{10}$ Similarly, when new patients were admitted, outgoing residents reported providing detailed information to the receiving resident including the medical history and the main reason for hospitalization, current diagnosis, and planned diagnostic tests. When a resident completes a block rotation (e.g., 1 month) as the primary provider, they give a detailed handoff to the new resident beginning the rotation.

\section{DISCUSSION}

Ensuring safety, effectiveness, and continuity of care during handoffs is a main concern for hospital-based care providers. We found that handoffs involved both explicit actions and implicit assumptions made by residents in transferring rights, duties, and obligations to one another. Successfully negotiating handoff interactions also required that residents balance relational factors to match content and dynamic risk. Residents were found to use recipient design during handoffs across three sites and study participants. First, they attempted to adjust the level, amount, and type of information shared based on the perceived or known preferences of the receiving resident, which might include interpersonal characteristics (i.e., reputation and/or training level). Second, some tailoring practices stemmed from the micro culture and the division of labor on a particular service (i.e., medicine or surgery). Third, residents reported that they adjusted their communication based on a recipient-designed assessment of situational factors that included the receiving residents' workload, time, training background, seniority, familiarity with patients, and patient acuity.

Our findings are consistent with research showing that a receiving resident's reputation or competence, informed by past performance, is taken into account during handoffs. ${ }^{11}$ The amount and type of information transferred is geared to the presumed training level of the receiver. For example, less experienced residents often include more irrelevant information that lengthens the handoff. ${ }^{39}$ Among dozens of handoff training programs, I-PASS is the only program that incorporates attention to context and contingency planning as standard considerations. ${ }^{40}$ Yet, these studies lack focus on the range of resident thought processes and the many adaptations that underlie how communication is tailored "on the fly" during handoffs. This 
First Generation

- Unidirectional flow of information

- Mechanical transfer of rights, duties and obligations

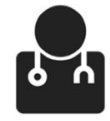

Hand off party
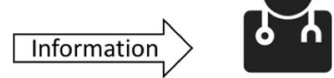

Receiver

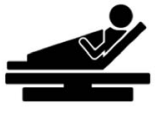

Patient

\section{Second Generation}

- Complex interactions involving reciprocal influence (recipient design)

- Recurring habits based on informal norms and cultures

- Repeated interactions lead to durable practices

- Interruptions, environment are barriers to effective interactions
Situational Context

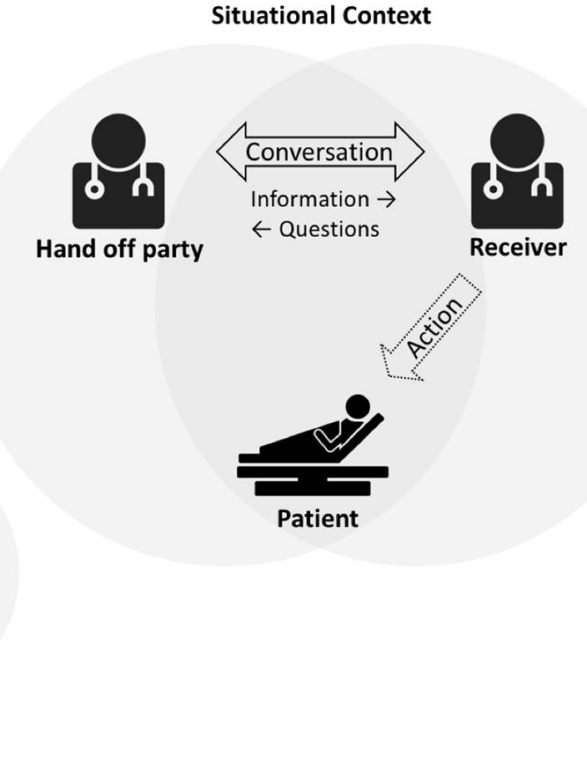

\section{Third Generation}

(1) Contextual influences

- Available technologies

(1) Situational Context

- Local culture

- $\quad$ Shift (Day, Night, etc.)

- Available time

- Handoff purpose

- Specialty

(2) Hand off parties
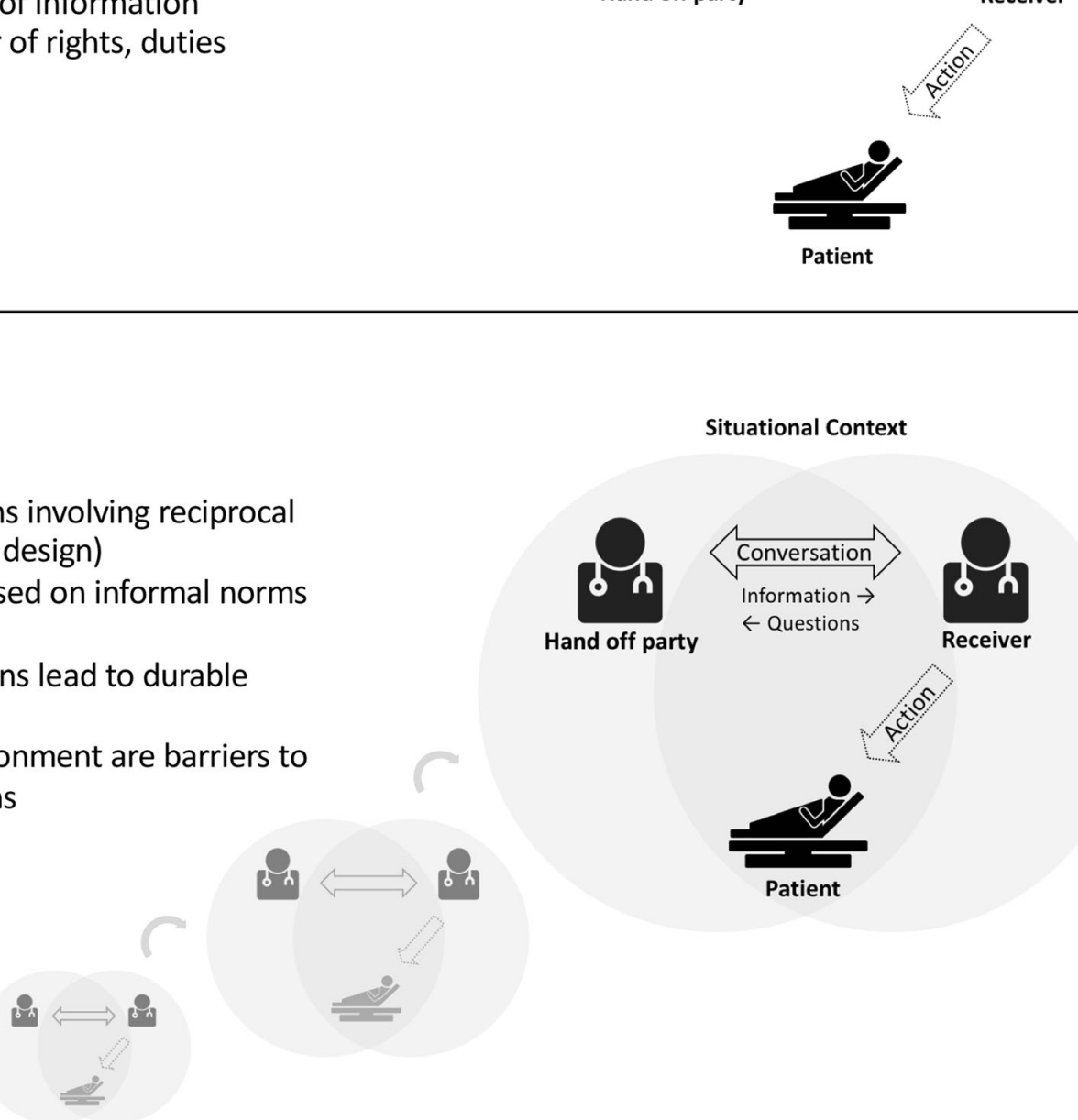

- Multiple physicians

- Unpredictable order of handoffs

(3) Receiver characteristics

- training level

- reputation

- preferences

- Actions

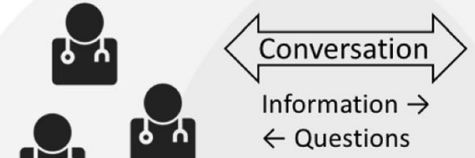

ton

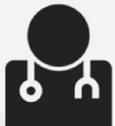

(3) Receiver

(2) Hand off parties

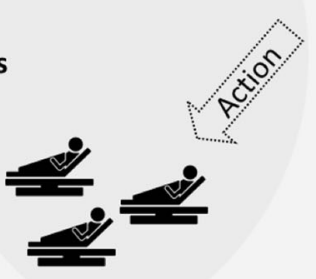

(4) Patients

(4) Patients

- Variable number and acuity of patients

Figure 1 Risk management typology of patient handoffs (HOP = handing off party). Adapted from Hilligoss and Cohen ${ }^{16}$. 
highlights why traditional interventions to improved handoffs have had only marginal success in improving effectiveness. ${ }^{7}$

Handoffs offer an opportunity for residents to contribute to the overall resilience of their team by being attuned to the timing and duration of the handoff in a contextually sensitive way. ${ }^{11}$ A recent study found that night to day shift handoffs focused on patients' active problems and were brief, whereas day to night shift handoffs were longer and consistently included patients' active problems, cause for admission, medical history, contingency plans, and previously completed tasks. ${ }^{41 \text {, }}$

${ }^{42}$ Residents offer foresight, coping strategies, and ideas about how to recover from eventual patient safety risks and deterioration. ${ }^{43}$ In practical terms, residents make anticipatory management judgments about which receiving physicians need specific "if/then" statements or contingency plans. ${ }^{21}$ Residents sought to bolster resilience and error detection and correction by providing more details, allowing for longer handoffs when the receiving resident was junior, patients had higher acuity, or the reputation of the resident warranted it.

Figure 1 is an emerging typology of three models for managing patient handoffs. The first diagram depicts handoffs as unilateral information exchanges. Second-generation solutions depict handoffs as multi-functional routines shaped by questions from receiving residents related to contextual factors affecting all parties. ${ }^{8}$ Our research extends this model by underscoring how handoffs are tailored in a dynamic manner using several dimensions in time and space to the specific residents' strengths and limitations, individual characteristics, available technologies ${ }^{13}$ and their work shift or departmental culture. ${ }^{44}$ This model offers an innovative way to account for how residents think about, enact, and reflect on handoffs. Understanding the moment-by-moment social construction of handoffs provides insights into the informal workplace culture that might a standardized approach might miss.

Well-known interventions address situational factors such as level of training, time on rotation, and knowledge of patients. ${ }^{40}$ Standardized approaches such as computer-generated patient lists from electronic medical record data and mnemonic-driven practices embedded into the current generation of handoff practices are also helpful but fall short. ${ }^{45,}{ }^{46}$ Notably, residents still tailor these "standard" artifacts by folding them, writing on them, and communicating information in unpredictable and inconsistent ways. ${ }^{47}$ Standardized solutions that do not account for these local innovations and improvisations in resident sense-making will likely only partially succeed. ${ }^{48}$ Designing agile, contextsensitive clinical procedures can enable receiving residents to better accomplish the tasks they face. Overemphasis on standardizing information transfer and responsibility functions of handoffs risks neglecting the critical role that tacit knowledge and adaptation plays in protecting patients and ongoing enculturation for trainees. ${ }^{49-51}$ Key differences between day-to-night, night-to-day, and double handoffs suggest that innovative training is needed to prepare residents for different handoff types. ${ }^{41}$

Our study has several limitations. Although participants included internal medicine and surgical residents, we lacked an adequate number of residents for statistically meaningful comparisons between specialties. Nevertheless, information provided by individual residents in both groups helped us generate a comprehensive picture of how residents tailored their actions. CTA methodology yields self-reported evidence rather than direct observations of patient handoff-tailoring behavior and is subject to social desirability bias. ${ }^{52}$

\section{CONCLUSIONS}

Clinical handoffs are complex social interactions based on implicit workplace norms and understanding. Residents use multiple cognitive strategies to adjust the levels and types of information they provide during handoffs. Recipient design practices are often based on tacit informal knowledge as well as the time and function of the handoff. Interventions designed to improve patient handoffs should more explicitly account for the fundamental role of recipient design in achieving reliable, safe patient care.

Corresponding Author: Nicholas A. Rattray, PhD; VA HSR\&D Center for Health Information and Communication Roudebush VAMC, Indianapolis, USA (e-mail: nrattray@iupui.edu).

Authors' Contribution All authors were involved in the design of this research, participated in manuscript development, and critically revised the manuscript for its intellectual content. RMF obtained study funding and directed the study. NAR, MEF, LGM, PB, ZF, PE, and RMF participated in data analysis. NAR, RMF, MEF, and LGM drafted the manuscript, and NAR, MEF, RMF, LGM, ZF, PE, PB, SUR, and HSG read, revised, and approved the final version. We appreciate the efforts of Paige DeChant in data collection and analysis, and Dr. Maddamsetti Rao, Christopher Kurtz, Ava Harms, Angela Kuramoto, Naomi Ashlely, and Natalia Skorohod for assistance in recruitment and logistics. We thank Rachel Gruber for excellent assistance with manuscript preparation and submission, and Julie Dilulio for her graphic design contribution. We would also like to thank the VA residents that volunteered to take time out of their regular duties to participate in the study.

Funding The research was funded by the Center for Health Information and Communication, Department of Veterans Affairs (VA), Veterans Health Administration, Health Services Research and Development Service (CIN 13-416), Project No. IIR 12-090.

\section{Compliance with Ethical Standards:}

Ethics approval was obtained from the University Institutional Review Board and the VAMC R\&D Human Subjects board.

Conflict of Interest: The authors declare that they do not have a conflict of interest.

Publisher's Note: Springer Nature remains neutral with regard to jurisdictional claims in published maps and institutional affiliations.

\section{REFERENCES}

1. Kitch BT, Cooper JB, Zapol WM, et al. Handoffs causing patient harm: a survey of medical and surgical house staff. Jt Comm J Qual Saf 2008;34(10):563-570.

2. Horwitz LI, Moin T, Krumholz HM, Wang L, Bradley EH. Consequences of inadequate sign-out for patient care. Arch Intern Med 2008;168(16):1755-1760. 
3. Arora VM, Manjarrez E, Dressler DD, Basaviah P, Halasyamani L, Kripalani S. Hospitalist handoffs: a systematic review and task force recommendations. J Hosp Med 2009;4(7):433-440.

4. Riesenberg LA, Leitzsch $\mathbf{J}$, Massucci JL, et al. Residents' and attending physicians' handoffs: a systematic review of the literature. Acad Med 2009;84(12):1775-1787.

5. Kripalani S, LeFevre F, Phillips CO, Williams MV, Basaviah P, Baker DW. Deficits in communication and information transfer between hospital-based and primary care physicians: implications for patient safety and continuity of care. JAMA 2007;297(8):831-841.

6. Hesselink G, Schoonhoven $\mathbf{L}$, Barach $\mathbf{P}$, et al. Improving patient handovers from hospital to primary care: a systematic review. Ann Intern Med 2012;157(6):417-428.

7. Hesselink G, Vernooij-Dassen M, Pijnenborg L, et al. Organizational culture: an important context for addressing and improving hospital to community patient discharge. Med Care 2013;51(1):90-98.

8. Cohen MD, Hilligoss PB. The published literature on handoffs in hospitals: deficiencies identified in an extensive review. Qual Saf Health Care 2010;19(6):493-497.

9. Hilligoss B, Mansfield JA, Patterson ES, Moffatt-Bruce SD. Collaborating-or "selling" patients? a conceptual framework for emergency department-to-inpatient handoff negotiations. Jt Comm J Qual Saf 2015;41(3): 134-143.

10. Toccafondi G, Albolino S, Tartaglia R, et al. The collaborative communication model for patient handover at the interface between high-acuity and low-acuity care. BMJ Qual Saf 2012;21 Suppl 1:i58-66.

11. Hilligoss B, Cohen MD. Hospital handoffs as multifunctional situated routines: implications for researchers and administrators. Advances in Health Care Management 2011;11:91-132.

12. Patterson ES, Wears RL. Patient handoffs: standardized and reliable measurement tools remain elusive. Jt Comm J Qual Saf 2010;36(2):5261.

13. Laugaland $\mathbf{K}$, Aase $\mathbf{K}$, Barach $\mathbf{P}$. Interventions to improve patient safety in transitional care-a review of the evidence. Work 2012;41 Suppl 1:2915-2924.

14. Tannen D, Wallat C. Doctor/mother/child communication: linguistic analysis of a pediatric interaction. In: Fisher S, Todd AD, eds. The Social Organization of Doctor-Patient Communication. Washington, D.C. Center for Applied Linguistics; 1983:203-220.

15. Maynard DW, Heritage J. Conversation analysis, doctor-patient interaction and medical communication. MEDU Medical Education 2005;39(4):428-435.

16. West C. Routine complications : Troubles with talk between doctors and patients. Bloomington: Indiana University Press; 1984.

17. Frankel RM. From sentence to sequence: understanding the medical encounter through microinteractional analysis. Discourse Processes 1984;7(2):135-170.

18. Sacks H, Schegloff EA, Jefferson G. A Simplest Systematics for the Organization of Turn-Taking for Conversation. Language 1974;50(4):696735.

19. Goodwin C. Conversational organization: interaction between speakers and hearers. New York: Academic Press; 1981.

20. Coulter J. The Sacks lectures. Hum Stud 1995;18(2/3):327-336.

21. Bergman AA, Flanagan ME, Ebright PR, O'Brien CM, Frankel RM. "Mr Smith's been our problem child today...": anticipatory management communication (AMC) in VA end-of-shift medicine and nursing handoffs. BMJ Qual Saf 2016;25(2):84-91.

22. Patterson ES, Roth EM, Woods DD, Chow R, Gomes Jo. Handoff strategies in settings with high consequences for failure: lessons for health care operations. Int J Qual Health Care 2004;16(2):125-132.

23. Collins SA, Mamykina L, Jordan D, et al. In search of common ground in handoff documentation in an intensive care unit. J Biomed Inform 2012;45(2):307-315.

24. Arora V, Johnson J. A model for building a standardized hand-off protocol. Jt Comm J Qual Saf 2006;32(11):646-655

25. Hoffman RR, Militello LG. Perspectives on cognitive task analysis: historical origins and modern communities of practice. New York: Taylor and Francis; 2008.

26. Crandall B, Hoffman RR, Klein GA. Working minds: a practitioner's guide to cognitive task analysis. Cambridge, MA: MIT Press; 2006.

27. Militello L, Hutton $\mathbf{R}$. Applied cognitive task analysis (ACTA): a practitioner's toolkit for understanding cognitive task demands. Ergonomics 1998;41(11):1618-1641.

28. Katz AM, Shotter J. Hearing the patient's 'voice': toward a social poetics in diagnostic interviews. Soc Sci Med 1996;43(6):919-931.

29. Gilchrist v. Key informant interviews. In: Crabtree BF, Miller WL, eds. Doing qualitative research. Newbury Park, CA: Sage Publishers; 1992:7089.

30. Heckathorn DD. Snowball versus respondent-driven sampling. Sociol Methodol 2011;41(1):355-366.

31. Dominguez C, Hutton R, J. F, McKellar D. Perception-action coupling in endoscopic surgery: a cognitive task analysis approach In: Barry B, Boutsma R, Guiard Y, eds. Studies in perception and action III. Mawah N.J.: Lawrence Erlbaum Associates; 1995.

32. Crandall, Calderwood R. Clinical assessment skills of experienced neonatal intensive care nurses [Contract 1 R43 NR0191101 for the National Center for Nursing, NIH] Fairborn, OH: Klein Associates Inc.; 1989.

33. Christensen RE, Fetters MD, Green LA. Opening the black box: cognitive strategies in family practice. Ann Fam Med 2005;3(2):144-150.

34. Patterson MD, Militello LG, Bunger A, et al. Leveraging the critical decision method to develop simulation-based training for early recognition of sepsis. J Cogn Eng Decis Mak 2016;10(1):36-56.

35. Glazer B, Strauss A. The discovery of grounded theory. New York: Aldine; 1967.

36. Borkan J. Immersion crystallization. In: Crabtree BF, Miller WL, eds. Doing qualitative research, Second Edition. Thousand Oaks, CA: Sage; 1999:179-194.

37. Lincoln Y, Guba E. Naturalistic inquiry. Newbury Park: Sage; 1985.

38. Guest G, MacQueen KM, Namey EE. Applied thematic analysis. Los Angeles: Sage Publications; 2012.

39. Cohen MD, Hilligoss PB. Handoffs in hospitals: a review of the literature on information exchange while transferring patient responsibility or control. Qual Saf Health Care 2010. 19(6):493-7

40. Starmer AJ, Spector ND, Srivastava R, Allen AD, Landrigan CP, Sectish TC. I-pass, a mnemonic to standardize verbal handoffs. Pediatrics 2012;129(2):201-204.

41. Lee S-H, Desai SV, Phan PH. The impact of duty cycle workflow on signout practices: a qualitative studyof an internal medicine residency program in Maryland, USA. BMJ Open. 2017;7(5):e015762.

42. Cosman PH, Sirimanna P, Barach P. Building surgical expertise through the science of continuous learning and training. In: Sanchez JA, Barach P, Johnson JK, Jacobs JP, eds. Surgical patient care: improving safety, quality, and values: Springer Berlin; 2017.

43. Jeffcott SA, Ibrahim JE, Cameron PA. Resilience in healthcare and clinical handover. Qual Saf Health Care 2009;18(4):256-260.

44. Johnson JK, Barach P, Vernooij-Dassen M. Conducting a multicentre and multinational qualitative study on patient transitions. BMJ Qual Saf 2012;21 Suppl 1:i22-28.

45. Young JQ, Ten Cate O, O'Sullivan PS, Irby DM. Unpacking the complexity of patient handoffs through the lens of cognitive load theory. Teach Learn Med 2016;28(1):88-96.

46. Riesenberg LA, Leitzsch J, Little BW. Systematic review of handoff mnemonics literature. Am J Med Qual 2009;24(3):196-204.

47. Militello LG, Rattray NA, Flanagan ME, et al. "Workin' on Our Night Moves": how residents prepare for shift handoffs. Jt Comm J Qual Patient Saf. 2018;44(8):485-493.

48. Barach P, Phelps G. Clinical sensemaking: a systematic approach to reduce the impact of normalised deviance in the medical profession. J R Soc Med 2013;106(10):387-390.

49. Stiles BM, Reece TB, Hedrick TL, et al. General surgery morning report: a competency-based conference that enhances patient care and resident education. Curr Surg 2006;63(6):385-390.

50. Williams RG, Silverman R, Schwind C, et al. Surgeon information transfer and communication: factors affecting quality and efficiency of inpatient care. Ann Surg 2007;245(2):159-169.

51. Wolf $\mathbf{Z R}$. Nursing rituals. The Canadian journal of nursing research $=$ Revue canadienne de recherche en sciences infirmieres 1988;20(3):59-69.

52. Orne MT. On the social psychology of the psychological experiment: with particular reference to demand characteristics and their implications. Am Psychol 1962;17(11):776-783. 